\section{Case Reports in Oncology}

Case Rep Oncol 2019;12:513-522

DOI: $10.1159 / 000501713$

Published online: July 10, 2019

(C) 2019 The Author(s)

Published by S. Karger AG, Basel

www.karger.com/cro

This article is licensed under the Creative Commons Attribution-NonCommercial 4.0 International License (CC BY-NC) (http://www.karger.com/Services/OpenAccessLicense).

Usage and distribution for commercial purposes requires written permission.

\title{
Preservation of the Epiphysis and Growth Plate in the Surgical Management of Femoral Osteosarcoma in a Skeletally Immature Patient by Intercalary Resection and Biological Reconstruction: A Case Report
}

\author{
Yuhei Yoda ${ }^{a, b}$ Sayaka Iwai Yamaguchi ${ }^{a}$ Toru Hirozane $^{a}$ \\ Naofumi Asano ${ }^{a}$ Atsuhito Seki ${ }^{b}$ Hideo Moriokac Robert Nakayama ${ }^{a}$ b \\ Masaya Nakamura ${ }^{a}$ Morio Matsumoto ${ }^{a}$ \\ aDepartment of Orthopaedic Surgery, Keio University School of Medicine, Tokyo, Japan; \\ ${ }^{b}$ Department of Orthopaedic Surgery, National Center for Child Health and Development, \\ Tokyo, Japan; 'Department of Orthopedic Surgery, Tokyo Medical Center, Tokyo, Japan
}

\section{Keywords}

Childhood osteosarcoma $\cdot$ Limb salvage $\cdot$ Biological reconstruction $\cdot$ Frozen tumor-devitalized autograft

\begin{abstract}
Osteosarcoma arises most frequently in the metaphysis around the knee and its management by limb salvage surgery in skeletally immature pediatric patients is extremely challenging. Common reconstructive methods such as endoprosthetic or biological reconstruction are not fully capable of dealing with durability-related and growth-related problems and their functional outcomes are not as good as those seen in adult cases. A definitive limb salvaging procedure in children that outperforms amputation or rotationplasty has not yet been established. Herein, we report a case of stage IV osteosarcoma in the femur of a 7-year-old boy that was safely managed with intercalary resection preserving the distal femoral growth plate and epiphysis, followed by biological reconstruction using a frozen tumor-devitalized autograft.
\end{abstract}


Good response to preoperative chemotherapy and the diaphyseal location of the tumor enabled us to perform a tumor resection that spared the growth plate and preserved the native knee joint structure. Plate fixation over the growth plate was terminated by removing the locking screws in the epiphysis after 44 months, which restored growth capacity to some extent. At 50 months postoperatively, no recurrence or progression of the disease was observed. The patient uses an extension shoe and reports having little discomfort in his daily life despite having a restricted range of motion and limb length discrepancy. In conclusion, limb salvage with biological reconstruction in skeletally immature patients can provide an acceptable functional outcome, including minimized limb length discrepancy, if critical damage to the growth plate and articular components can be avoided.

\section{Introduction}

Osteosarcoma (OS) is the most common primary malignant tumor of the bone in children [1]. With appropriate wide resection and adjuvant chemotherapy, more than $70 \%$ of cases are thought to be curable and therefore functional prognosis after tumor resection has emerged as a current topic of discussion [2]. Limb salvage has become a major surgical option in many cases and the development of reconstructive methods using endoprostheses or biological materials has markedly improved patient outcomes [2]. However, treating skeletally immature pediatric patients with such reconstructive methods is enormously challenging, especially in patients younger than 10 years old. First, the size of the remaining bone can be too small for endoprosthetic reconstruction. Second, even when the remaining bone is large enough for endoprosthetic reconstruction, there is a greater possibility of future implant failure in younger patients. The limited durability of endoprostheses results in aseptic loosening or mechanical failure and the risk is multiplied over a patient's lifetime [2-4]. Therefore, in pediatric cases biological reconstruction is preferred as a permanent solution when the adjacent joint is preserved after tumor resection. However, even with this alternative considerable limb length discrepancy cannot be avoided [3-5] because the growth plate needs to be resected en-bloc with the tumor in a great majority of patients with metaphyseal osteosarcoma in the long bones $[2,6]$. Extendable endoprostheses and distraction osteogenesis are common methods used to prevent growth-related problems. Even so, repetitive surgical interventions are required in these procedures, which escalates the risk of complications, such as infection, which could cause functional impairment [4]. Accordingly, in skeletally immature OS patients, limb salvage is not always the better option compared with amputation and rotationplasty, especially when such problems discussed above occur [4]. More insights and technical advances are required for the improvement of limb salvaging surgeries in childhood OS.

Here we report a case of femoral OS in a 7-year-old skeletally immature patient, treated with intercalary resection and reconstruction with a tumor-devitalized autograft treated with liquid nitrogen freezing to preserve his native knee joint and adjacent growth plate.

\section{Case Presentation}

A 7-year-old boy with a history of hereditary retinoblastoma (Rb) and autism was referred to our hospital complaining of left knee pain and gait disturbance. On physical examination, his left thigh was painful and hot on palpation and swollen to $32.6 \mathrm{~cm}$ in girth compared with a right thigh girth measurement of $30.9 \mathrm{~cm}$. An X ray of the left femur revealed 
sclerotic change in the diaphysis, accompanied by a local periosteal reaction (Fig. 1a), which suggested a malignant bone tumor. MRI showed a massive bone tumor that measured $14 \times$ $3 \times 4 \mathrm{~cm}$ extending from the diaphysis to the distal metaphysis, which was hypointense on T1weighted images, heterogeneously hyperintense on T2-weighted images and enhanced after gadolinium administration (Fig. 1b-d). An extraosseous mass was also observed. A CT scan showed a small nodule in the lung suggestive of a pulmonary metastasis (Fig. 1e), and an incisional biopsy led to the diagnosis of OS, stage IV (T2N0M1a).

He received three courses of neoadjuvant chemotherapy with adriamycin, cisplatin and high-dose methotrexate. The preoperative imaging studies showed a satisfactory response to chemotherapy; ossification of the extraosseous lesion, decreased levels of gadolinium enhancement and shrinkage of the lung nodule (Fig. 2a-c).

The surgical procedure comprised a wide resection and reconstruction using a frozen tumor-bearing autograft. Using a longitudinal curved incision on the lateral side, the surgical approach began between the rectus femoris and vastus lateralis and proceeded around the tumor leaving vastus intermedius as the adequate margin for the wide resection. Then an osteotomy was carried out along the Kirschner wires which were placed as cutting guide under intraoperative fluoroscopy reserving a margin of $2 \mathrm{~cm}$ (Fig. 2d, e). The distal osteotomy line was carefully positioned just above the physis. Next, after the tumor and attached soft tissues were completely removed, the resected bone was subjected to a freezing procedure in liquid nitrogen for 20 minutes as previously described (Fig. 2f) [7]. Then the thawed devitalized bone graft was repositioned and a non-vascularized free fibula autograft was inserted in the proximal junction. A distal femur locking plate was used for a rigid fixation (Fig. 2g). The tumor specimen was examined histologically and the response to neoadjuvant chemotherapy was evaluated as Grade 3 with 90\% tumor necrosis.

Postoperatively, following 3 weeks in a long leg cast and wheelchair the patient was allowed to walk with an ischial weight-bearing knee-ankle-foot orthosis (KAFO). Postoperative chemotherapy was reinitiated on the 10th day after surgery, and surgery for the residual lung metastasis was carried out following completion of the chemotherapy.

Twelve months after the primary surgery, the distal part of the frozen graft showed a radiolucent appearance on an X ray suggestive of bone resorption of the autograft (Fig. 3a). Lowintensity pulsed ultrasound (LIPUS) was immediately started and the patient was instructed to avoid weight bearing with the use of a KAFO. Taking these precautions, we safely precluded any further failure and union was observed after 12 months of the additional treatment (Fig. $3 b$ ). To allow longitudinal growth, screws in the distal femoral epiphysis were removed at 44 months postoperatively (Fig. 3c). At this point the locking plate had migrated proximally from the original position and the epiphyseal plate remained open on an X-ray.

Fifty months after the initial surgery, at the age of 11, the spina-malleolar distance of the affected limb was $30 \mathrm{~mm}$ shorter than the contralateral side. Despite the fact that there was a measured difference in leg lengths, growth at the preserved distal growth plate of the femur could be seen as the locking plate had migrated slightly further in the proximal direction during the 6 months after screw removal (Fig. 3d). Valgus deformity was also observed with a femorotibial angle of $168^{\circ}$ on the operated side and $174^{\circ}$ on the other. There was no difference in tibial length. Although the range of motion of the knee was restricted to $0-45^{\circ}$, the patient did not experience any severe inconvenience in his daily activities. Slight limping was observed but he managed to walk independently with an extension shoe without pain. At the latest visit, his Musculoskeletal Tumor Society (MSTS) functional score [8] was 23/30 (77\%). Neither recurrence nor metastasis has been observed so far. 


\section{Discussion}

We experienced a case of OS in the femur of a 7-year-old boy, which we managed by way of knee joint sparing surgery with biological reconstruction that preserved the growth plate. Leg length discrepancy and functional disability has been handled to above an acceptable degree. Although the patient was initially diagnosed as stage IV OS with pulmonary metastasis, an excellent oncologic outcome has been maintained for 50 months postoperatively.

Limb salvaging procedures are currently often opted for in extremity OS cases as advances in chemotherapy and preoperative imaging studies have enabled us to perform precisely-planned minimal surgical resections that avoid excessive functional loss [2, 9]. Oncologic outcomes in such cases are reported to be almost the same as those after amputations, as long as the patient responds well to chemotherapy [10]. Although consensus has not yet been obtained, the appropriate margin for resection of an OS with a good response to chemotherapy has been discussed. Further, no correlation has been reported between surgical margin width and local recurrence (1-10 mm vs. 11-20 mm) [11]. For epiphyseal-sparing resection such as ours, the distance between joint articular cartilage and the tumor is recommended to be $20 \mathrm{~mm}$ at minimum, securing at least $10 \mathrm{~mm}$ margin width from the tumor and $10 \mathrm{~mm}$ thickness in the residual epiphysis $[9,10]$. In our case, the most distal location of the tumor which was $2 \mathrm{~cm}$ proximal to the growth plate, and the efficacy of preoperative chemotherapy enabled us to preserve his knee joint by way of intercalary resection, despite the fact that the bone tumor extended quite far longitudinally, with nearly $2 / 3$ of the femur having to be resected. The distal osteotomy was carefully placed just above the growth plate with the aim of minimizing any damage.

An optimal reconstructive technique is also essential to achieve successful limb salvage. When considering reconstructive procedures after bone tumor resection around the knee, the first step in decision making is to consider whether the native joint structure can be preserved [4]. Biological reconstruction procedures, such as allograft, distraction osteogenesis, vascularized fibular graft and tumor-devitalized autograft, tend only to be applicable in joint-preservable cases, while endoprostheses are chosen in patients whose affected epiphyses need to be resected en-bloc [4]. The patient's age is another important factor. Biological reconstruction methods tend to be preferred in limb salvage surgeries in younger patients, despite the fact that prostheses are advantageous in their strength, which permits earlier mobilization and weight-bearing [3-7]. This preference is mainly because of the high potential for later complications following prosthetic replacement related to durability, which could end up with a patient needing major revision surgery. Among the established biological reconstructive methods, tumor-devitalized autografts are preferred in Japan, as allografts are not as available as in other countries due to social and religious beliefs [7]. Irradiation, autoclaving, pasteurization and hypothermic procedures using liquid nitrogen have been introduced to kill tumor cells for recycling resected bone grafts. Frozen tumor devitalization with liquid nitrogen was first reported in 2005 and accumulating evidence has since supported its practicality [6, 7, 12, 13]. Advantages are the simplicity of the whole procedure, the provision of a perfect fit to the defect, preserving anchorage for soft tissue and ligamentous attachment, and restoring osteoinductive proteins within the bone. Reported complications are non-union, fractures, infection and recurrence of the tumor in surrounding soft tissue. Local recurrence in the treated bone has never been reported $[6,7,12,13]$. Several retrospective studies on OS cases treated with joint sparing resection and frozen tumor-devitalized autografts have proved its applicability for juxtaarticular lesions, even in weight-bearing joints $[6,12,13]$. Favorable functional outcomes were obtained in these studies, with mean MSTS scores achieving $90 \%$ of the 
perfect score. Recently, its possible applicability as a growth-preserving method in skeletally immature patients has been suggested [6]. An analysis of epiphyseal growth after this type of surgery in 12 cases of childhood OS around the knee (follow-up period; 41-90 months) was conducted and it was found that transverse growth of the epiphysis was conserved in all cases. This finding is important as growth disturbance after limb salvaging surgeries around the knee in skeletally immature pediatric patients has always been considered as being inevitable and repetitive surgical interventions to correct length inequality or deformity can impair a patient's long-term quality of life or functional outcome [3-6]. In accordance with these findings, and considering our patient's age and tumor location, we reconstructed the defect with a frozen tumor-devitalized autograft. In our particular case, the fixation over the physis was arranged to be temporary with the intention of allowing natural longitudinal growth of the distal femur to occur. During the postoperative course, however, transient resorption of the autograft was observed and its union was delayed, thus additional treatment with LIPUS was necessary. Consequently, epiphyseal screw removal for freeing the physis was postponed until 44 months postoperatively and limb shortening was observed at the latest follow-up. We could only find one report referring to a similar procedure in which epiphyseal screws were removed after 18 months and it also described such limitations [6]. This implies that there might be factors other than the prolonged period of fixation that compromise skeletal growth and further studies are required to understand the mechanism. However, minimizing damage to the growth plate seems to have been beneficial in our case as the locking plate that initially bridged the epiphysis and diaphysis migrated away from the distal femoral epiphysis, implying the presence of a viable growth plate. As the patient is expected to undergo a growth spurt over the coming years, we assume the preserved growth capacity, albeit partially damaged, might hopefully obscure the current length inequality during this period. Careful observation will be needed until skeletal maturity. Currently, with the use of an extension shoe the patient claims to have little inconvenience in his daily life and we recognize no necessity for any further intervention. Functionally, the outcome of our case has not been as good as other cases in previous reports $[12,13]$, mainly resulting from the restricted range of motion of the knee joint. We assess its cause to be the delayed engraftment of the autograft, which meant he had to use a KAFO for as long as two years and the difficulty in providing effective physiotherapy due to his age and autism diagnosis. Joint mobilization procedures, distraction osteogenesis and correction osteotomy for the valgus deformity should be considered in the future when he begins to experience more difficulties in his daily life.

Lastly, the prognosis of OS secondary to $\mathrm{Rb}$ is reported to be the same or even better than that of general OS cases, as long as complete resection of the tumor is possible and chemotherapeutic response is good [14]. With the fact that both the primary and metastatic OS lesions were totally resectable and the patient responded fairly well to the chemotherapy, we regard our patient's prognosis to be as such. No recurrence or metastasis has been found in the 50 months following the primary surgery. However strict follow-up on generation of another new malignancy is essential in such hereditary Rb cases. Among survivors of this genetically-caused tumor syndrome, long-term survival is sometimes terminated by a second neoplasm and the overall incidence of more than one second malignancy is reported to be $6 \%$ in hereditary Rb survivors [15]. 


\section{Conclusion}

Stage IV osteosarcoma of the femoral diaphysis in a 7-year-old skeletally immature patient was surgically managed with joint preserving resection and frozen tumor-bearing autograft reconstruction in combination with chemotherapy and surgical removal of the lung metastasis. Despite the successful preservation of the knee joint, including the growth plate and epiphysis, limb length discrepancy and functional disability occurred to some extent, however the patient claims to have little discomfort in his daily life 50 months after surgery. Careful observation, especially of future growth, is needed considering his age and the technically restored growth capacity of the femur.

\section{Statement of Ethics}

The authors have no ethical conflicts to disclose. Informed consent was obtained from the patient's parent for publication of this case report and the accompanying images.

\section{Disclosure Statement}

The authors have no conflicts of interest to declare.

\section{Author Contributions}

All authors performed the collection and assembly of the data. YY performed the data analysis and prepared the initial draft of the manuscript. YY, SIY and RN contributed to the writing of the manuscript. RN provided final approval of the manuscript. All authors have read and approved the final manuscript.

\section{References}

1 Fletcher C, Bridge J, Hogendoorn P, Mertens F. WHO classification of tumours of soft tissue and bone. 4th ed. Lyon: IARC Press; 2013.

2 Muscolo DL, Ayerza MA, Aponte-Tinao LA, Ranalletta M. Partial epiphyseal preservation and intercalary allograft reconstruction in high-grade metaphyseal osteosarcoma of the knee. J Bone Joint Surg Am. 2004 Dec;86(12):2686-93.

3 Levin AS, Arkader A, Morris CD. Reconstruction following tumor resections in skeletally immature patients. J Am Acad Orthop Surg. 2017 Mar;25(3):204-13.

4 Morris CD, Wustrack RL, Levin AS. Limb-salvage options in growing children with malignant bone tumors of the lower extremity: a critical analysis review. JBJS Rev. 2017 Jul;5(7):e7.

5 Aponte-Tinao LA, Albergo JI, Ayerza MA, Muscolo DL, Ing FM, Farfalli GL. What are the complications of allograft reconstructions for sarcoma resection in children younger than 10 years at long-term followup? Clin Orthop Relat Res. 2018 Mar;476(3):548-55.

6 Takeuchi A, Yamamoto N, Hayashi K, Matsubara H, Kimura H, Miwa S, et al. Growth of epiphysis after epiphyseal-preservation surgery for childhood osteosarcoma around the knee joint. BMC Musculoskelet Disord. 2018 Jun;19(1):185.

7 Tsuchiya H, Wan SL, Sakayama K, Yamamoto N, Nishida H, Tomita K. Reconstruction using an autograft containing tumour treated by liquid nitrogen. J Bone Joint Surg Br. 2005 Feb;87(2):218-25.

8 Enneking WF, Dunham W, Gebhardt MC, Malawar M, Pritchard DJ. A system for the functional evaluation of reconstructive procedures after surgical treatment of tumors of the musculoskeletal system. Clin Orthop Relat Res. 1993 Jan;286(286):241-6. 
9 Aponte-Tinao L, Ayerza MA, Muscolo DL, Farfalli GL. Survival, recurrence, and function after epiphyseal preservation and allograft reconstruction in osteosarcoma of the knee. Clin Orthop Relat Res. 2015 May;473(5):1789-96.

10 Li J, Wang Z, Ji C, Chen G, Liu D, Zhu H. What are the oncologic and functional outcomes after joint salvage resections for juxtaarticular osteosarcoma about the knee? Clin Orthop Relat Res. 2017 Aug;475(8):2095104.

11 Andreou D, Bielack SS, Carrle D, Kevric M, Kotz R, Winkelmann W, et al. The influence of tumor- and treatment-related factors on the development of local recurrence in osteosarcoma after adequate surgery. An analysis of 1355 patients treated on neoadjuvant Cooperative Osteosarcoma Study Group protocols. Ann Oncol. 2011 May;22(5):1228-35.

12 Higuchi T, Yamamoto N, Nishida H, Hayashi K, Takeuchi A, Kimura H, et al. Knee joint preservation surgery in osteosarcoma using tumour-bearing bone treated with liquid nitrogen. Int Orthop. 2017 Oct;41(10):2189-97.

13 Igarashi K, Yamamoto N, Shirai T, Hayashi K, Nishida H, Kimura H, et al. The long-term outcome following the use of frozen autograft treated with liquid nitrogen in the management of bone and soft-tissue sarcomas. Bone Joint J. 2014 Apr;96-B(4):555-61.

14 Bielack SS, Kempf-Bielack B, Heise U, Schwenzer D, Winkler K; Cooperative German-Austrian-Swiss Osteosarcoma Study Group. Combined modality treatment for osteosarcoma occurring as a second malignant disease. J Clin Oncol. 1999 Apr;17(4):1164-74.

15 Wong J, Morton L, Tucker M, Abramson D, Seddon J, Sampson J, et al. Risk of subsequent malignant neoplasms in long-term hereditary retinoblastoma survivors after chemotherapy and radiotherapy. J Clin Oncol. 2014 Oct;32(29):3284-3290. 


\section{Case Reports in Oncology}

\begin{tabular}{l|l}
\hline Case Rep Oncol 2019;12:513-522 \\
\hline DOI: 10.1159/000501713 & $\begin{array}{l}\text { ( ) 2019 The Author(s). Published by S. Karger AG, Basel } \\
\text { www.karger.com/cro }\end{array}$ \\
\hline
\end{tabular}
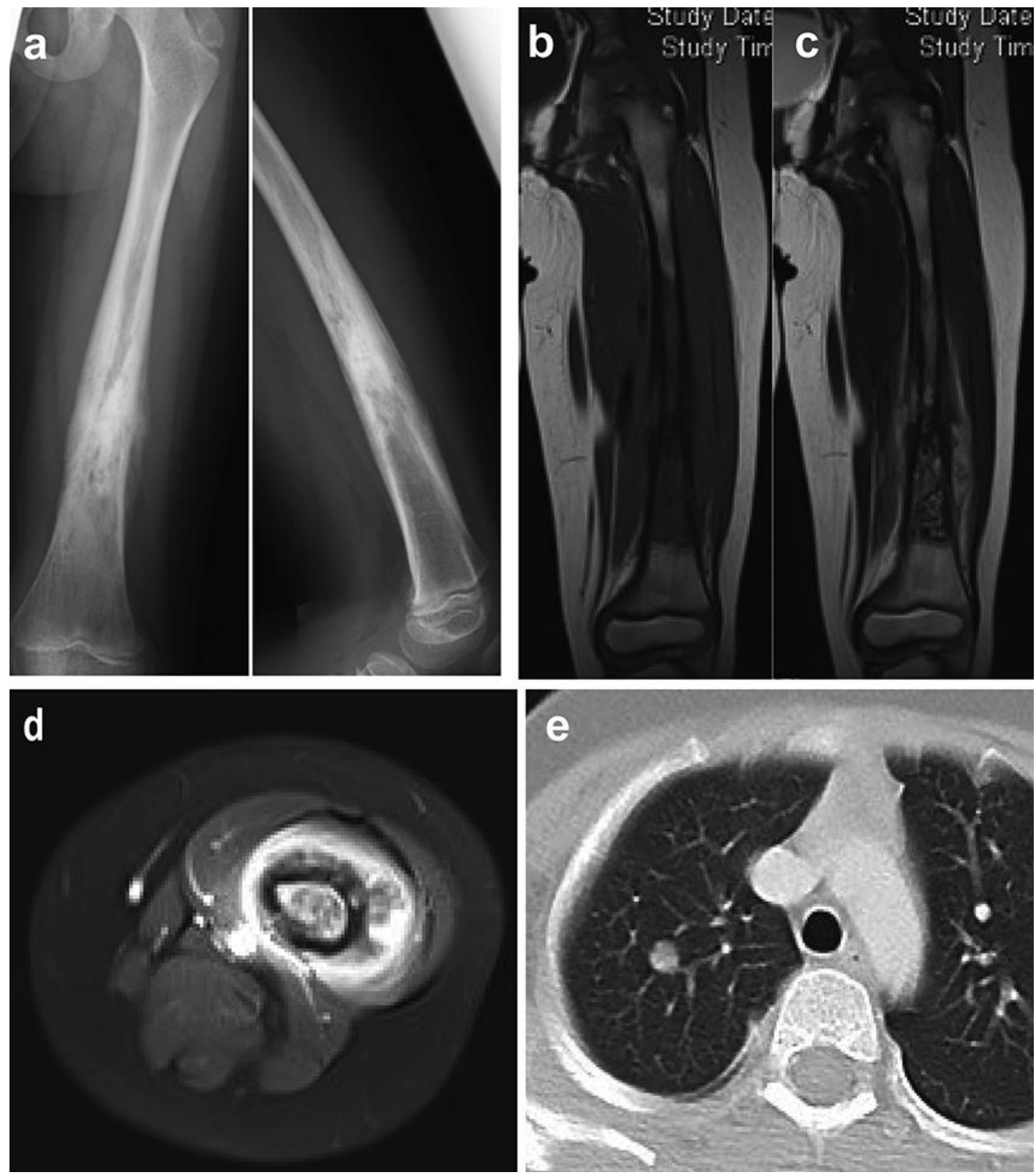

Fig. 1. X rays at the patient's first visit showed a sclerotic lesion in the femur accompanied by a periosteal reaction (a). MRI revealed a bone tumor with low intensity on T1-weighted images (b), and heterogeneous high intensity on T2-weighted images (c), which invaded extraosseously and was strongly enhanced with gadolinium (d). Pulmonary metastasis was observed on a CT scan (e). 


\section{Case Reports in Oncology}

\begin{tabular}{l|l}
\hline Case Rep Oncol 2019;12:513-522 \\
\hline DOI: 10.1159/000501713 & $\begin{array}{l}\text { @ 2019 The Author(s). Published by S. Karger AG, Basel } \\
\text { www.karger.com/cro }\end{array}$ \\
\hline
\end{tabular}

Yoda et al.: Biological Reconstruction Preserving the Growth Plate in Femoral OS of a Pediatric Patient
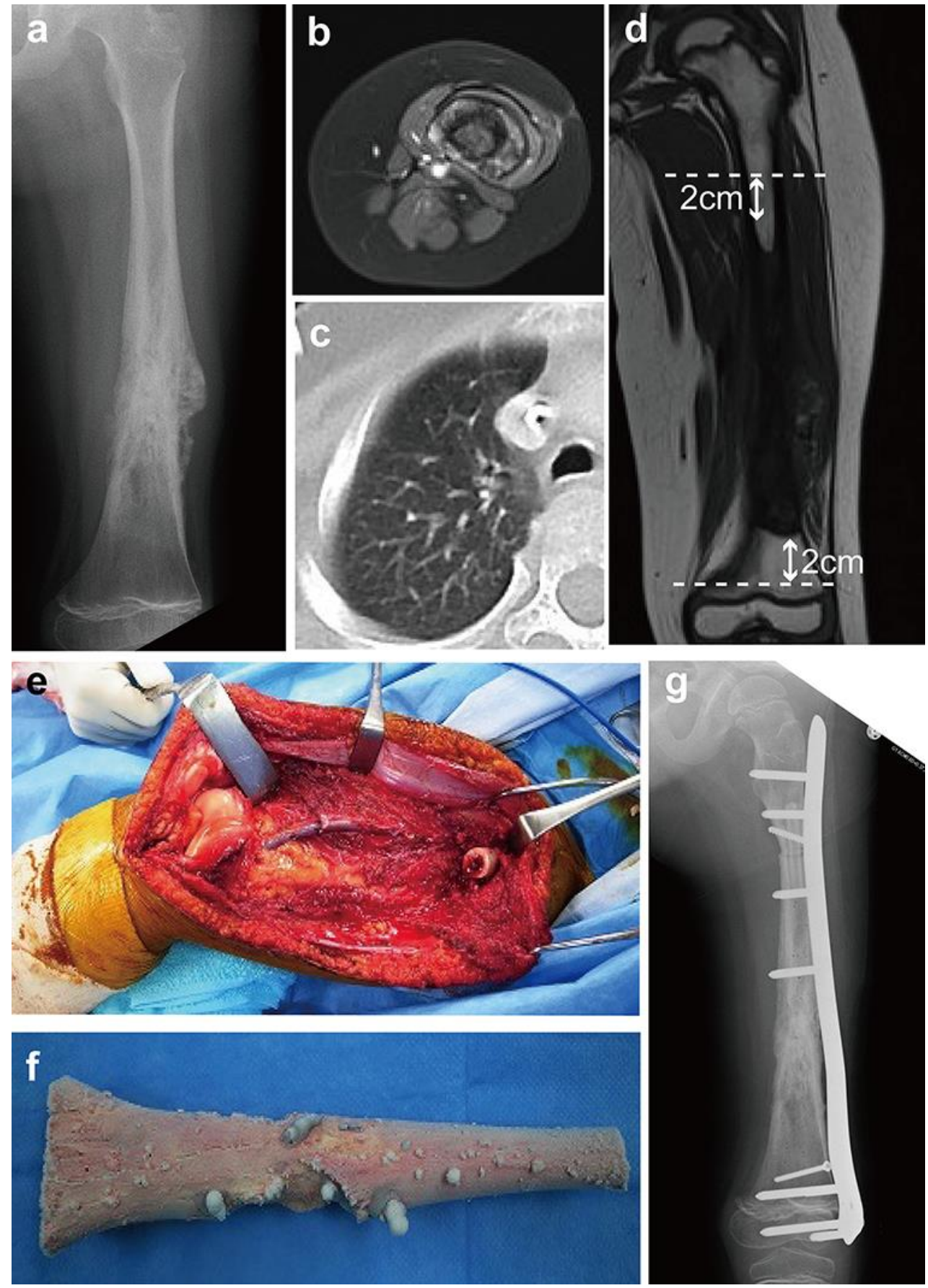

Fig. 2. Preoperative imaging findings confirmed the patient's good response to chemotherapy with ossification of the extraosseous lesion observed on an X ray (a), reduced extraosseous mass with weakened gadolinium intake on MRI (b) and shrinkage of the pulmonary nodule on a CT scan (c). During surgery, osteotomy was planned as indicated by dashed lines in (d), reserving surgical margin as shown by doubleheaded arrows. The defect after the tumor resection (e) was reconstructed with the frozen tumor-devitalized graft ( $f$ ) and firmly fixed with the use of a locking plate and intramedullary insertion of the free fibula autograft (g). 


\section{Case Reports in Oncology}
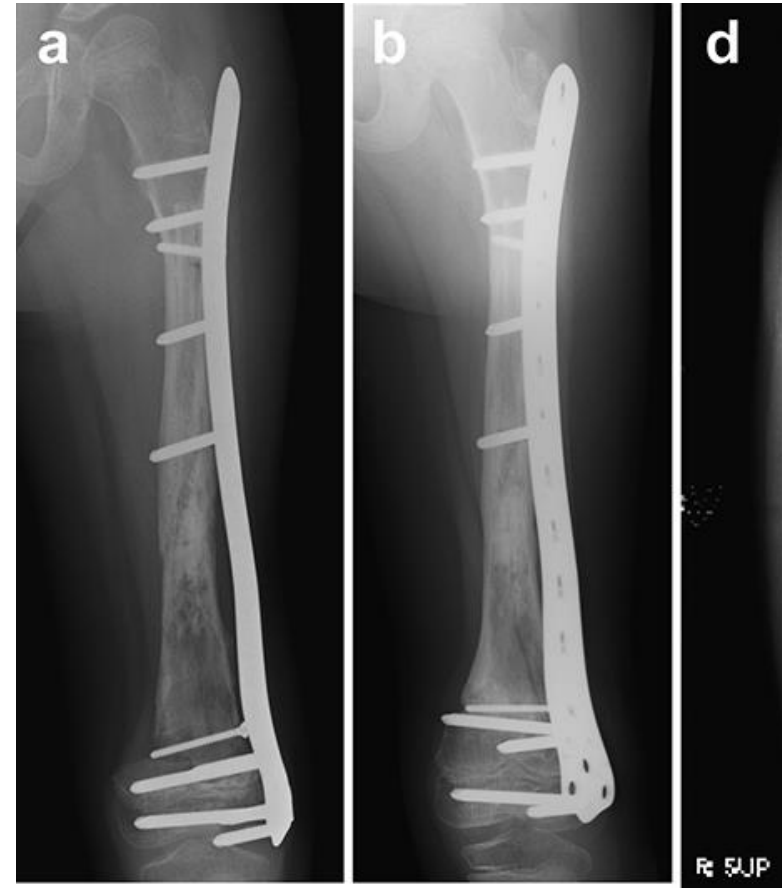

(c) 2019 The Author(s). Published by S. Karger AG, Basel www.karger.com/cro

Yoda et al.: Biological Reconstruction Preserving the Growth Plate in Femoral OS of a Pediatric Patient
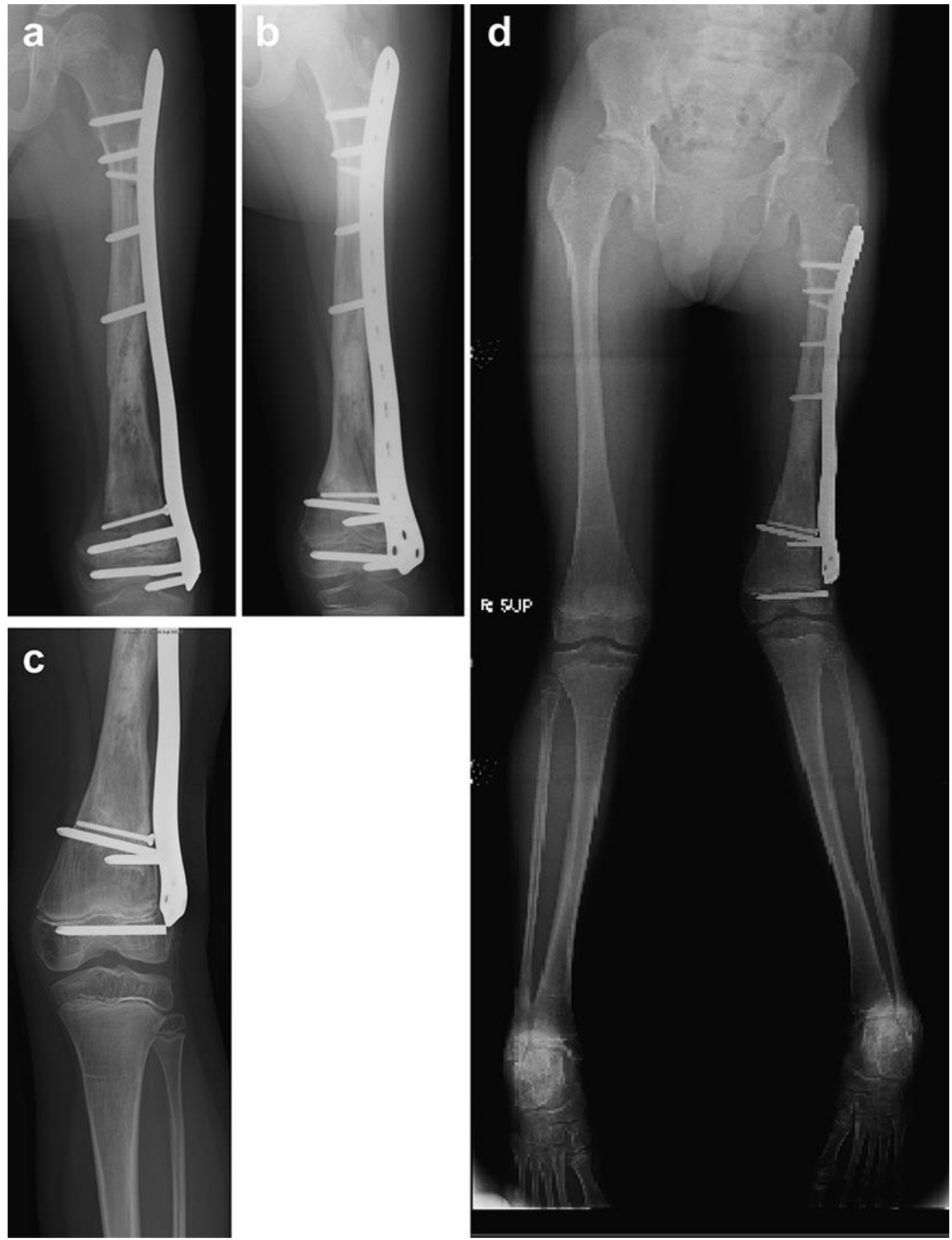

Fig. 3. At 12 months postoperatively (P.O.), resorptive change in the distal part of the graft was observed on an X ray (a), which was recovered with the assistance of LIPUS and avoiding weight by using a KAFO for 12 months (b). The distal locking screws were removed at 44 months P.O. after complete engraftment and union (c). An X ray at the latest visit, 50 months P.O. (d), revealed shortening by $43 \mathrm{~mm}$ of the affected limb, resulting from the growth disturbance of the femur and a valgus deformity. Proximal migration of the distal end of the locking plate in relation to the distal epiphysis was observed, suggesting viable growth capacity. 